\title{
A Theory of Baroclinic Turbulence
}

\author{
BRIAN F. FARRELL \\ Department of Earth and Planetary Sciences, Harvard University, Cambridge, Massachusetts \\ Petros J. IOANNOU \\ Department of Physics, National and Capodistrian University of Athens, Athens, Greece
}

(Manuscript received 22 October 2008, in final form 29 January 2009)

\begin{abstract}
Understanding the physical mechanism maintaining fluid turbulence remains a fundamental theoretical problem. The two-layer model is an analytically and computationally simple system in which the dynamics of turbulence can be conveniently studied; in this work, a maximally simplified model of the statistically steady turbulent state in this system is constructed to isolate and identify the essential mechanism of turbulence. In this minimally complex turbulence model the effects of nonlinearity are parameterized using an energetically consistent stochastic process that is white in both space and time, turbulent fluxes are obtained using a stochastic turbulence model (STM), and statistically steady turbulent states are identified using stochastic structural stability theory (SSST). These turbulent states are the fixed-point equilibria of the nonlinear SSST system. For parameter values typical of the midlatitude atmosphere, these equilibria predict the emergence of marginally stable eddy-driven baroclinic jets. The eddy variances and fluxes associated with these jets and the power-law scaling of eddy variances and fluxes are consistent with observations and simulations of baroclinic turbulence. This optimally simple model isolates the essential physics of baroclinic turbulence: maintenance of variance by transient perturbation growth, replenishment of the transiently growing subspace by nonlinear energetically conservative eddy-eddy scattering, and equilibration to a statistically steady state of marginal stability by a combination of nonlinear eddy-induced mean jet modification and eddy dissipation. These statistical equilibrium states provide a theory for the general circulation of baroclinically turbulent planetary atmospheres.
\end{abstract}

\section{Introduction}

Physical understanding of synoptic-scale baroclinic dynamics has been advanced by recognition of the role of nonnormality in producing perturbation growth and in determining the statistical structure of the eddy field. We refer to the ideas and methods by which stability theory is extended through incorporation of the effects of nonnormality as generalized stability theory (GST) (Farrell 1982, 1985, 1989; Farrell and Ioannou 1993b, 1994, 1996a,b). The method within GST for obtaining the statistics of the eddy field given a jet - the stochastic turbulence model (STM) (Farrell and Ioannou 1995, 1996a; DelSole and Farrell 1995, 1996; DelSole 1996;

Corresponding author address: Brian Farrell, Department of Earth and Planetary Sciences, Harvard University, Geological Museum 452, 24 Oxford Street, Cambridge, MA 02138.

E-mail: farrell@seas.harvard.edu
Newman et al. 1997; Whitaker and Sardeshmukh 1998; Zhang and Held 1999; DelSole 2004b) —is a building block for the recently developed theory for the structure of turbulent jets, namely, stochastic structural stability theory (SSST) (Farrell and Ioannou 2003, 2007, 2008, 2009). In this work we exploit these methods to further our understanding of the fundamental physics of turbulence.

We begin building an idealized but physically and mechanistically correct closure for turbulence by noting that the nonlinear terms in the hydrodynamic equations do not participate in the transfer of energy from the mean flow to the eddy field, so it follows that eddy variance is maintained by the linear perturbation terms in the dynamics. We anticipate, in accord with observations, that this linear process is nonmodal perturbation growth (DelSole 2004a, 2007). It follows that the key physical mechanism sustaining turbulence is that process maintaining the nonnormal subspace of growing perturbations 
against continual depletion under shearing by the mean flow. In statistically steady unforced turbulence, this process is the energetically conservative scattering by nonlinear wave-wave interaction; because this scattering process is chaotic and has short time and space scales, it can be parameterized as stochastic (DelSole 2004b; Sardeshmukh and Sura 2007).

Given sufficient excitation of the nonnormal growing subspace to maintain the observed turbulence in a jet, the detailed structure of the turbulence and the associated eddy fluxes can be obtained using an STM. With eddy forcing of the mean jet obtained from the STM, the nonlinear dynamical balance among the ensemble mean turbulent heat and momentum flux divergences, the mean thermal forcing, and the dissipation can be obtained using SSST (Farrell and Ioannou 2003, 2007). In the case of the Jovian winds, which are driven by internally generated convection (Gierasch et al. 2000; Ingersoll et al. 2000), the stochastic excitation needed to maintain the observed turbulence can be identified as that of the observed convection, so that in this system turbulence does not require a further closure (Farrell and Ioannou 2009). In the case of the midlatitude jet, although part of the excitation of the growing subspace is traceable to essentially chaotic convection, as in the Jovian case, a substantial portion originates in the nonlinear eddy-eddy scattering. One approach to account for the implicit nature of the eddy-eddy scattering is to obtain the equilibrium turbulent states for all plausible scattering intensities and then identify the physical operating point of the midlatitude turbulence, taking for the scattering parameterization that intensity producing the observed jet structure and eddy statistics (Farrell and Ioannou 2008). This approach has the advantage of simply and directly identifying the essential dynamics maintaining the turbulent statistical equilibrium state, but it falls short of a closure because the eddy-eddy scattering remains an explicit parameter.

To close the turbulence problem, the nonlinear eddyeddy scattering of energy among scales must be parameterized in a physically correct manner-that is, quadratic in velocity, stochastic in time and space, and conservative of energy. ${ }^{1}$ We construct a closure with these properties that is specified by a single scalar parameter controlling the scattering efficiency. Although the scattering efficiency remains a parameter, it could (at least in principle) be found from calculation of the wave-wave scattering coefficients in Fourier space

\footnotetext{
${ }^{1}$ The nonlinear scattering process conserves potential enstrophy as well as energy, but we consider potential enstrophy to be a weak invariant and do not insist that it be conserved.
}

(Tung and Orlando 2003; DelSole 2007). We discuss the physical meaning and effect of this scattering coefficient below.

Having obtained the excitation from the stochastic scattering parameterization and the fluxes from the STM, it remains to obtain the equilibrium statistically steady turbulent state. Study of midlatitude baroclinic jet dynamics reveals the mechanism of this equilibration to be wave-mean flow interaction producing fixed points of mutual adjustment between the turbulent eddy field and the mean jet structure (Farrell and Ioannou 2008). We use an SSST model to locate these equilibria in which the turbulent state is maintained in a statistical equilibrium with essentially correct physics. Baroclinic turbulence adjusts the atmospheric state to marginally stable equilibria and thus these equilibria constitute the basis for a theoretical understanding of the general circulation of turbulence-dominated circulations such as that of the midlatitude atmosphere.

We present two examples: turbulence supported by relaxation to a meridionally localized unstable temperature gradient, and turbulence supported in a wide channel relaxed to a meridionally constant unstable temperature gradient. In the first case, the equilibrium jet is found to be in good agreement with nonlinear model simulations of localized jets (DelSole and Farrell 1996; Zurita-Gotor 2007); in the second, multiple jets emerge and equilibrate in agreement with simulations of turbulence in wide baroclinic channels (Panetta 1993; Thompson and Young 2007).

An important problem into which these equilibria provide insight is that of determining the physical mechanism underlying the turbulent heat flux-thermal gradient relationship. Two primary mechanisms have been advanced to explain this relationship: baroclinic adjustment (Stone 1978) and turbulent diffusion (Green 1970; Held and Larichev 1996). The baroclinic adjustment hypothesis has been criticized for predicting fixed thermal gradients whereas the turbulent diffusion hypothesis has been criticized for failing to predict observed fluxgradient relationships (Zurita-Gotor 2007; Thompson and Young 2007). We find that as the thermal gradient is increased across a wide channel, at first multiple jets adjust to maintain marginally stable equilibria and the heat flux increases rapidly with the imposed thermal gradient until, at a sufficiently high imposed thermal gradient, jets are no longer maintained but the rapid increase of heat flux with thermal gradient persists. This behavior agrees with the concept of baroclinic adjustment in the sense that the system is modified from an unstable to a marginally stable state by eddy fluxes, but the process of adjustment produces a functional dependence between the flux and the gradient that varies 
to good approximation as a power law in agreement with predictions of higher-order thermal diffusion (Held and Larichev 1996; Pavan and Held 1996; Thompson and Young 2007).

Although the turbulent states obtained in this work are strikingly realistic when compared with turbulent states in nonlinear simulations, the goal of this work is to provide a fundamental understanding of the physical mechanisms of turbulence rather than to optimize comparison with model integrations. For this reason we have always chosen the simplest parameterization even if a nonessential improvement in turbulence structure could be obtained with a more complex parameterization. For example, we have chosen uniform Rayleigh damping to model eddy dissipation rather than variance dependent diffusion, which would improve our jet structure (DelSole and Farrell 1996), and we have chosen an excitation that is uniform and white in space and time to model eddy-eddy scattering rather than a variance-dependent scattering (DelSole 2001), which would improve the verisimilitude of the jet structure. We have made these choices to make clear that the form of the dissipation and the scattering parameterization used in the closure are not essential to the physics of turbulence; rather, that the fundamental mechanisms of turbulence are replenishment of the nonnormal growing subspace and nonlinear equilibration by jet structure modification.

\section{Dynamics of baroclinic turbulent jets}

\section{a. Formulation}

A theory of jet dynamics in turbulence was developed in Farrell and Ioannou (2003). This theory was applied to the problem of the formation of jets in barotropic turbulence in Farrell and Ioannou (2007), to the problem of formation of jets in baroclinic turbulence in Farrell and Ioannou (2008), and to the problem of the formation of jets in the shallow water equations on an equatorial beta plane in Farrell and Ioannou (2009). We now briefly review this theory as it applies to the baroclinic turbulence closure problem.

Consider a two-layer baroclinic fluid on a midlatitude $\beta$ plane. Quasigeostrophic motions are governed by the equation

$$
\begin{aligned}
& \frac{\partial q_{n}}{\partial t}+u_{n} \frac{\partial q_{n}}{\partial x}+v_{n} \frac{\partial q_{n}}{\partial y} \\
& =-r_{2} \nabla^{2} \psi_{i} \delta_{i 2}+(-1)^{n} 2 r_{R} \lambda^{2}\left(\Psi^{-}-\psi^{-}\right)
\end{aligned}
$$

where $n=1$ refers to the top layer and $n=2$ to the bottom layer. The potential vorticity is defined in terms of the upper- and lower-layer streamfunction $\psi_{n}$ as

$$
q_{n}=\nabla^{2} \psi_{n}+(-1)^{n} 2 \lambda^{2} \psi^{-}+\beta y,
$$

with the baroclinic (denoted -) and the barotropic $($ denoted +$)$ streamfunctions defined as

$$
\psi^{-}=\frac{\psi_{1}-\psi_{2}}{2} \quad \text { and } \quad \psi^{+}=\frac{\psi_{1}+\psi_{2}}{2} .
$$

The corresponding zonal $(x)$ and meridional $(y)$ velocities in each layer are

$$
u_{n}=-\frac{\partial \psi_{n}}{\partial y} \quad v_{n}=\frac{\partial \psi_{n}}{\partial x},
$$

with similar definitions for the barotropic and baroclinic velocities.

In (1), $r_{2}$ is the rate of damping of the mean flow in the lower layer (layer 2) to a state of rest (as indicated by the Kronecker $\delta_{i 2}$, which is nonzero only for the lower layer $i=2$ ). The baroclinic flow is relaxed at rate $r_{R}$ to the imposed baroclinic shear:

$$
U_{R}^{-}=-\Psi_{y}^{-} .
$$

The nondimensional Rossby radius of deformation is $1 / \lambda=\sqrt{g^{\prime} H} / f L$, where $L$ is the horizontal scale, $H$ is the height of each layer, $g^{\prime}=g\left(\rho_{2}-\rho_{1}\right) /\left(\rho_{2}+\rho_{1}\right)$ is the reduced gravity ( $g$ is the gravitational acceleration and $\rho_{n}$ the density of the layers), and $f$ is the Coriolis parameter. ${ }^{2}$

Lengths are nondimensionalized by $L=10^{6} \mathrm{~m}$ and time by the Earth day $t_{d}=1$ day so that the velocity scale is $L / t_{d}=11.6 \mathrm{~m} \mathrm{~s}^{-1}$. All variables will henceforth be considered nondimensional. In all calculations $\lambda^{2}=1$.

Fields are decomposed into zonal averages and perturbations:

$$
\psi_{n}=\overline{\psi_{n}}+\psi_{n}^{\prime}
$$

in which a bar denotes the zonal average. Averaging (1), we obtain the equation for the mean

$$
\begin{aligned}
\frac{\partial \overline{q_{n}}}{\partial t}= & -r_{2} \nabla^{2} \overline{\psi_{i}} \delta_{i 2}+(-1)^{n} 2 r_{R} \lambda^{2}\left(\Psi^{-}-\overline{\psi^{-}}\right) \\
& -\overline{\left(u_{n}^{\prime} \frac{\partial q_{n}^{\prime}}{\partial x}+v_{n}^{\prime} \frac{\partial q_{n}^{\prime}}{\partial y}\right)}
\end{aligned}
$$

and by subtraction the equation for the perturbations:

$$
\begin{aligned}
\frac{\partial q_{n}^{\prime}}{\partial t}+ & \overline{u_{n}} \frac{\partial q_{n}^{\prime}}{\partial x}+v_{n}^{\prime} \frac{\partial \overline{q_{n}}}{\partial y} \\
= & -r_{2} \nabla^{2} \psi_{i}^{\prime} \delta_{i 2}-(-1)^{n} 2 r_{R} \lambda^{2} \psi^{-\prime} \\
& +\frac{\partial}{\partial x}\left(u_{n}^{\prime} q_{n}^{\prime}-\overline{u_{n}^{\prime} q_{n}^{\prime}}\right)+\frac{\partial}{\partial y}\left(v_{n}^{\prime} q_{n}^{\prime}-\overline{v_{n}^{\prime} q_{n}^{\prime}}\right) .
\end{aligned}
$$

\footnotetext{
${ }^{2}$ The two-level interpretation equivalently sets the static stability $N=\left(g^{\prime} / H\right)^{1 / 2}$, so that $1 / \lambda=N H / f L$.
} 
The quadratic eddy-eddy interaction term on the rhs of (7) is parameterized as stochastic excitation and augmentation to the rate of eddy dissipation:

$$
\begin{aligned}
& \frac{\partial}{\partial x}\left(u_{n}^{\prime} q_{n}^{\prime}-\overline{u_{n}^{\prime} q_{n}^{\prime}}\right)+\frac{\partial}{\partial y}\left(v_{n}^{\prime} q_{n}^{\prime}-\overline{v_{n}^{\prime} q_{n}^{\prime}}\right) \\
& \quad \approx \iint W_{n}\left(x-x^{\prime}, y-y^{\prime}\right) \eta_{n}\left(x^{\prime}, y^{\prime}, t\right) d x^{\prime} d y^{\prime}-r_{e} q_{n}^{\prime},
\end{aligned}
$$

where the integral is over the flow domain, $\eta_{n}(x, y, t)$ is a variable random in space and time with zero mean and unit variance such that

$$
\left\langle\eta_{n}(x, y, t) \eta_{m}\left(x^{\prime}, y^{\prime} t^{\prime}\right)\right\rangle=\delta_{n m} \delta\left(x-x^{\prime}\right) \delta\left(y-y^{\prime}\right) \delta\left(t-t^{\prime}\right),
$$

$r_{e}$ is a rate of eddy dissipation with the form of Rayleigh friction, and \langle\rangle indicates an ensemble average. The function $W_{n}$ sets both the amplitude of the stochastic excitation and its spatial distribution (Farrell and Ioannou 1993a, 1996a; DelSole and Farrell 1996; Newman et al. 1997; DelSole 2004b). The excitation could be exogenous, as is appropriate for the gaseous planets, or partially exogenous to account for, say, random excitation by midlatitude cumulus events, but in this work we will assume that only endogenous forcing that is quadratic in the eddy amplitude occurs. Further, because the nonlinear terms do not provide net energy to the eddy field but only redistribute energy among perturbations, the energy input from the stochastic excitation will be balanced by an augmentation of the uniform Rayleigh friction to enforce energy conservation.

Under the ergodic assumption, the zonal mean can be replaced by the ensemble mean:

$$
\overline{u_{n}^{\prime} q_{n}^{\prime}}=\left\langle u_{n}^{\prime} q_{n}^{\prime}\right\rangle, \quad \overline{v_{n}^{\prime} q_{n}^{\prime}}=\left\langle v_{n}^{\prime} q_{n}^{\prime}\right\rangle .
$$

The ensemble mean of all quadratic eddy statistics can be obtained from the ensemble mean eddy covariance $\mathbf{C}$.

The barotropic and baroclinic perturbation streamfunctions are written as a Fourier sum of zonal harmonics:

$$
\psi^{ \pm}(x, y, t)=\sum_{k} \psi_{k}^{ \pm}(y, t) e^{i k x} .
$$

Note that the dash is dropped from perturbation quantities and (6) and (7) are discretized in the meridional so that continuous field variables in $y$ become column vectors collocated on equally spaced points $y_{i}$ separated in $y$ by distance $\delta$. Under the assumption that $\eta_{n}$ is a white noise process, ${ }^{3}$ the covariance matrix $\mathbf{C}_{k}$ associ-

\footnotetext{
${ }^{3}$ This is not a restriction because red noise processes can be easily incorporated; cf. Farrell and Ioannou (2009).
}

ated with the $k$ zonal wavenumber eddy fields satisfies the equation

$$
\frac{d \mathbf{C}_{k}}{d t}=\mathbf{A}_{k}(\mathbf{U}) \mathbf{C}_{k}+\mathbf{C}_{k} \mathbf{A}_{k}^{\dagger}(\mathbf{U})+\epsilon \frac{T \sqrt{E}}{E_{0}^{3 / 2}} \mathbf{Q}_{k}-r_{e} \mathbf{C}_{k},
$$

where is $E$ the total eddy energy, $T$ is the kinetic eddy energy, $E_{0}$ is a unit normalizing factor with the dimensions of energy per unit mass so that $T \sqrt{E} / E_{0}^{3 / 2}$ is dimensionless, $\epsilon$ is the scalar scattering coefficient, and $\mathbf{Q}_{k}$ is a covariance matrix associated with the spatial correlation $W_{n}$ in (8). In (12) $\mathbf{A}_{k}(\mathbf{U})$ is the operator of the dynamics linearized about the zonal mean flow $\mathbf{U}$, where

$$
\mathbf{U} \equiv\left(\begin{array}{l}
U^{+} \\
U^{-}
\end{array}\right)
$$

with barotropic and baroclinic components $U^{+}$and $U^{-}$.

The coefficient $r_{e}$ is specified by the requirement that the total energy introduced by the forcing be balanced by dissipation. Let the energy per unit mass associated with $\mathbf{C}_{k}$ be $E_{k}=\operatorname{trace}\left(\mathbf{M}_{k} \mathbf{C}_{k}\right)$, where $\mathbf{M}_{k}$ is the total energy metric [defined in (19)]. In order that the parameterized nonlinear terms do not contribute net energy, it is required that

$$
\epsilon \frac{E \sqrt{T}}{E_{0}^{3 / 2}} \sum_{k} \operatorname{trace}\left(\mathbf{M}_{k} \mathbf{Q}_{k}\right)=r_{e} E,
$$

with $E=\Sigma_{k} E_{k}$ and $T=\Sigma_{k} T_{k} ; T_{k}$ is the eddy kinetic energy at wavenumber $k$ given by $T_{k}=\operatorname{trace}\left(\mathbf{T}_{k} \mathbf{C}_{k}\right)$, where $\mathbf{T}_{k}$ is the eddy kinetic energy metric. The coefficient of the eddy-induced dissipation is

$$
r_{e}=\epsilon \sqrt{T} \frac{\sum_{k} \operatorname{trace}\left(\mathbf{M}_{k} \mathbf{Q}_{k}\right)}{E_{0}^{3 / 2}} .
$$

We choose to express the covariance $\mathbf{C}_{k}$ in terms of the barotropic and baroclinic streamfunction:

$$
\mathbf{C}_{k}=\left[\begin{array}{cc}
\mathbf{C}_{k}^{+} & \mathbf{C}_{k}^{ \pm} \\
\left(\mathbf{C}_{k}^{ \pm}\right)^{\dagger} & \mathbf{C}_{k}^{-}
\end{array}\right],
$$

with $\quad \mathbf{C}_{k}^{+}=\left\langle\psi_{k}^{+} \psi_{k}^{+\dagger}\right\rangle, \quad \mathbf{C}_{k}^{-}=\left\langle\psi_{k}^{-} \psi_{k}^{-\dagger}\right\rangle, \quad$ and $\quad \mathbf{C}_{k}^{ \pm}=$ $\left\langle\psi_{k}^{+} \psi_{k}^{-\dagger}\right\rangle$. In these variables the linear operator in (12) is

$$
\mathbf{A}_{k}(\mathbf{U})=\left[\begin{array}{ll}
\mathbf{A}_{k}^{+}\left(\mathbf{U}^{+}\right) & \mathbf{A}_{k}^{+}\left(\mathbf{U}^{-}\right) \\
\mathbf{A}_{k}^{-}\left(\mathbf{U}^{+}\right) & \mathbf{A}_{k}^{-}\left(\mathbf{U}^{-}\right)
\end{array}\right]
$$

in which the individual linear operators are

$$
\mathbf{A}_{k}^{+}\left(\mathbf{U}^{+}\right)=\boldsymbol{\Delta}^{-1}\left[-i k \mathbf{U}^{+} \boldsymbol{\Delta}-i k\left(\beta \mathbf{I}-\mathbf{D}^{2} \mathbf{U}^{+}\right)\right]-\frac{r_{2}}{2} \mathbf{I},
$$




$$
\begin{aligned}
\mathbf{A}_{k}^{+}\left(\mathbf{U}^{-}\right)= & \boldsymbol{\Delta}^{-1}\left(-i k \mathbf{U}^{-} \boldsymbol{\Delta}+i k \mathbf{D}^{2} \mathbf{U}^{-}\right)+\frac{r_{2}}{2} \mathbf{I}, \\
\mathbf{A}_{k}^{-}\left(\mathbf{U}^{-}\right)= & \left(\boldsymbol{\Delta}-2 \lambda^{2} \mathbf{I}\right)^{-1}\left[-i k \mathbf{U}^{-}\left(\boldsymbol{\Delta}+2 \lambda^{2} \mathbf{I}\right)\right. \\
& \left.+i k \mathbf{D}^{2} \mathbf{U}^{-}+\frac{r_{2}}{2} \boldsymbol{\Delta}\right], \text { and } \\
\mathbf{A}_{k}^{-}\left(\mathbf{U}^{+}\right)= & \left(\boldsymbol{\Delta}-2 \lambda^{2} \mathbf{I}\right)^{-\mathbf{1}}\left[-i k \mathbf{U}^{+}\left(\boldsymbol{\Delta}-\mathbf{2} \lambda^{2} \mathbf{I}\right)\right. \\
& \left.+i k\left(\beta \mathbf{I}-\mathbf{D}^{2} \mathbf{U}^{+}\right)-\frac{r_{2}}{2} \boldsymbol{\Delta}+2 r_{R} \lambda^{2} \mathbf{I}\right]
\end{aligned}
$$

with $\boldsymbol{\Delta} \equiv \mathbf{D}^{2}-k^{2} \mathbf{I}$ being the Laplacian and $\boldsymbol{\Delta}^{-1}$ the inverse Laplacian, with the appropriate lateral boundary conditions incorporated, and $\mathbf{I}$ is the identity matrix.

The forcing matrix $\mathbf{Q}_{k}$ is chosen to be

$$
\mathbf{Q}_{k}=\left|\alpha_{k}\right|^{2}\left(\begin{array}{cc}
\mathbf{W} \mathbf{W}^{\dagger} & 0 \\
0 & \mathbf{W} \mathbf{W}^{\dagger}
\end{array}\right),
$$

implying independent stochastic excitation of the barotropic and baroclinic streamfunction each delta correlated in time and meridionally correlated by $\mathbf{W}$. We select W matrices producing Gaussian autocorrelation of the excitation with width $\delta$ in $y$ about the latitude of excitation, $y_{i}$ : $\exp \left[-\left(y-y_{i}\right)^{2} / \delta^{2}\right]$ which, given that $\delta$ is the distance between points in $y$, corresponds to a continuous approximation to delta-correlated excitation in $y$. This minimal smoothing of the delta correlation of the excitation in $y$ is done for the purpose of improving the numerics and the results converge for sufficiently small values of $\delta$. The scalar coefficient $\alpha_{k}$ is chosen so that each perturbation wavenumber is forced with equal energy. The energy input associated with $\mathbf{Q}_{k}$ is given by trace $\left(\mathbf{M}_{k} \mathbf{Q}_{k}\right)$, with $\mathbf{M}_{k}$ being the total energy metric:

$$
\mathbf{M}_{k}=-\frac{1}{4}\left[\begin{array}{cc}
\Delta & 0 \\
0 & \left(\Delta-2 \lambda^{2} \mathbf{I}\right)
\end{array}\right]
$$

defined so that $\left(\psi_{k}^{+}, \psi_{k}^{-}\right) \mathbf{M}_{k}\left(\psi_{k}^{+}, \psi_{k}^{-}\right)^{\dagger}$ is the total energy per unit mass of state $\left(\psi_{k}^{+}, \psi_{k}^{-}\right)$at zonal wavenumber $k$. The kinetic energy metric is

$$
\mathbf{T}_{k}=-\frac{1}{4}\left(\begin{array}{cc}
\Delta & 0 \\
0 & \Delta
\end{array}\right)
$$

The metric that produces the eddy available potential energy is $\mathbf{M}_{k}-\mathbf{T}_{k}$.

The equation for the zonal mean flow [(6)] can be written in terms of the barotropic and baroclinic components:

$$
\frac{d \mathbf{U}}{d t}=\mathbf{G U}+\mathbf{H}\left(\mathbf{C}_{k}\right),
$$

in which $\mathbf{G}$ is the linear dynamical operator:

$$
\mathbf{G}=\left(\begin{array}{ll}
\mathbf{G}_{11} & \mathbf{G}_{12} \\
\mathbf{G}_{21} & \mathbf{G}_{22}
\end{array}\right)
$$

with components

$$
\begin{aligned}
& \mathbf{G}_{11}=-\frac{r_{2}}{2} \mathbf{I}, \\
& \mathbf{G}_{12}=\frac{r_{2}}{2} \mathbf{I}, \\
& \mathbf{G}_{21}=\left(\mathbf{D}^{2}-2 \lambda^{2} \mathbf{I}\right)^{-1}\left(\frac{r_{2}}{2} \mathbf{D}^{2}\right), \text { and } \\
& \mathbf{G}_{22}=\left(\mathbf{D}^{2}-2 \lambda^{2} \mathbf{I}\right)^{-1}\left(-\frac{r_{2}}{2} \mathbf{D}^{2}+2 r_{R} \lambda^{2} \mathbf{I}\right),
\end{aligned}
$$

and the mean flow forcing $\mathbf{H}\left(\mathbf{C}_{k}\right)$ is composed of the eddy forcing and the mean thermal relaxation toward the radiative equilibrium thermal wind $\boldsymbol{U}_{R}^{-}$:

$$
\mathbf{H} \equiv\left[\begin{array}{c}
\left\langle\mathbf{F}^{+}\right\rangle \\
\left(\mathbf{D}^{2}-2 \lambda^{2} \mathbf{I}\right)^{-1}\left(\mathbf{D}^{2} \mathbf{F}^{-}-2 r_{R} \lambda^{2} \boldsymbol{U}_{R}^{-}\right)
\end{array}\right] .
$$

The eddy forcing of the mean flow is expressed in terms of the barotropic and baroclinic eddy streamfunction under the assumption of ergodicity (DelSole and Farrell 1996; Valis 2006) as

$$
\begin{aligned}
& \left\langle F^{+}\right\rangle=\overline{\psi_{x}^{+} \psi_{y y}^{+}+\psi_{x}^{-} \psi_{y y}^{-}} \text {and } \\
& \left\langle F^{-}\right\rangle=\overline{\psi_{x}^{+} \psi_{y y}^{-}+\psi_{x}^{-} \psi_{y y}^{+}-2 \lambda^{2} \psi_{x}^{+} \psi^{-}} .
\end{aligned}
$$

The overbars denote zonal averaging. Using the property that the zonal average $\overline{a b}$ of the product of two sinusoidally varying fields $\hat{a} e^{i k x}$ and $\hat{b} e^{i l x}$ is $\operatorname{Re}\left(\hat{a} \hat{b}^{*}\right) \delta_{k l} / 2$ (* denotes the complex conjugate), we can express the eddy forcings [(26a) and (26b)] in terms of the eddy streamfunction Fourier amplitudes as

$$
\begin{aligned}
& \left\langle F^{+}\right\rangle=-\sum_{k} \frac{k}{2} \operatorname{Im}\left(\psi_{k}^{+} \mathbf{D}^{2} \psi_{k}^{+*}+\psi_{k}^{-} \mathbf{D}^{2} \psi_{k}^{-*}\right) \quad \text { and } \quad(27 \mathrm{a}) \\
& \left\langle F^{-}\right\rangle=-\sum_{k} \frac{k}{2} \operatorname{Im}\left(\psi_{k}^{+} \mathbf{D}^{2} \psi_{k}^{-*}+\psi_{k}^{-} \mathbf{D}^{2} \psi_{k}^{+*}-2 \lambda^{2} \psi_{k}^{+} \psi_{k}^{-*}\right),
\end{aligned}
$$

which can be expressed in terms of the covariances as

$$
\begin{aligned}
\left\langle\mathbf{F}^{+}\right\rangle & =\sum_{k}-\frac{k}{2} \operatorname{diag}\left[\operatorname{Im}\left(\mathbf{C}_{k}^{+}+\mathbf{C}_{k}^{-}\right) \mathbf{D}^{2 \dagger}\right], \quad \text { and } \\
\left\langle\mathbf{F}^{-}\right\rangle & =\sum_{k}-\frac{k}{2} \operatorname{diag}\left\{\operatorname{Im}\left[\left(\mathbf{C}_{k}^{ \pm}+\mathbf{C}_{k}^{ \pm \dagger}\right) \mathbf{D}^{2 \dagger}-2 \lambda^{2} \mathbf{C}_{k}^{ \pm}\right]\right\} .
\end{aligned}
$$


In summary, the ensemble mean turbulent system consisting of the eddies and the associated mean flow is governed by the autonomous deterministic equations:

$$
\begin{aligned}
\frac{d \mathbf{C}_{k}}{d t} & =\mathbf{A}_{k}(\mathbf{U}) \mathbf{C}_{k}+\mathbf{C}_{k} \mathbf{A}_{k}^{\dagger}(\mathbf{U})+\epsilon \frac{E \sqrt{T}}{E_{0}^{3 / 2}} \mathbf{Q}_{k}-r_{e} \mathbf{C}_{k}, \\
\frac{d \mathbf{U}}{d t} & =\mathbf{G U}+\mathbf{H}\left(\mathbf{C}_{k}\right),
\end{aligned}
$$

in which

$$
\begin{aligned}
& E=\sum_{k} \operatorname{trace}\left(\mathbf{M}_{k} \mathbf{C}_{k}\right), \quad T=\sum_{k} \operatorname{trace}\left(\mathbf{T}_{k} \mathbf{C}_{k}\right), \text { and } \\
& r_{e}=\epsilon \sqrt{T} \frac{\sum_{k} \operatorname{trace}\left(\mathbf{M}_{k} \mathbf{Q}_{k}\right)}{E_{0}^{3 / 2}} .
\end{aligned}
$$

This system is the minimal mechanistically correct turbulence model. In this system, energetically conservative scattering by eddy-eddy interaction is parameterized as white in space and time to within requirements of the numerics. This system is globally stable and the attractor set for midlatitude jet parameter values consists of fixed-point equilibria, although at other parameter values limit cycles are found.

\section{b. Scaling of the equations, boundary conditions, and parameters}

Solutions of (29) were found for a periodic channel of width $L_{y}=12$ in the meridional in the case of the radiatively forced localized jet with both baroclinic and barotropic shear. Solutions were also obtained for a channel width $L_{y}=24$ for the case of a meridionally uniform radiatively forced baroclinic jet. For the meridionally uniform jet, the size of the channel was selected to be wide enough to accommodate a number of jets; however, quantization effects were seen in some cases. The calculations used 64-point discretization over 12 meridional units in the meridional. In the zonal direction 14 waves are retained, consisting of global zonal wavenumbers 1 to 14 in a reentrant zonal channel of nondimensional length $L_{x}=40$.

For diagnostic purposes the meridional mean radiative equilibrium baroclinic shear $U_{R}^{-}$is related to the temperature difference across the channel by the thermal wind:

$$
\Delta T=\frac{2 f L_{y}}{R} U_{R}^{-} .
$$

\section{Results}

\section{a. Turbulent equilibration of a meridionally localized jet}

Consider the case of the radiative equilibrium Gaussian jet:

$$
U_{R}^{-}=U_{0}^{-} \exp \left[-\frac{\left(y-L_{y} / 2\right)^{2}}{4}\right] .
$$

Turbulence is maintained when the temperature difference across the channel with meridional width $L_{y}=12$ exceeds $\Delta T_{c}=11.75$, which coincides with the modal stability boundary.

The inverse eddy-induced dissipation rate $r_{e}$, defined in (15), can be identified with eddy turnover time. In the following calculations we assume the nondimensional values $E_{0}=1$ and $\Sigma_{k} \operatorname{trace}\left(\mathbf{M}_{k} \mathbf{Q}_{k}\right)=0.4389$. We anticipate $0.1<r_{e}<1.0$, corresponding to replenishment of perturbations on a time scale of days [cf. Tung and Orlando (2003), especially their Fig. 5, which reports energy injection rates as a function of wavenumber]. The eddy field comprises global zonal wavenumbers 1-14 and the damping parameters are $r_{2}=1 / 5$ day $^{-1}, r_{R}=1 / 10$ day $^{-1}$.

Turbulent equilibria are not sensitive to small changes in $\epsilon$ in the range $0.1<\epsilon<1$. For small scattering coefficients $(\epsilon<0.1)$, the equilibria give way to limit cycles and at $\epsilon=0$ there is no excitation and for small supercriticality we recover the vacillation regime described by Pedlosky $(1972,1977)$ and Pedlosky and Frenzen (1980).

The structure of the equilibrium jets, together with associated fluxes and energy spectra, are shown in Fig. 1 for $\epsilon=0.5$ and $\Delta T=35 \mathrm{~K}$ over the $12 \times 10^{3}-\mathrm{km}$ channel. The jet is equilibrated to a marginally stable state, despite a change of sign of the upper- and lower-level potential vorticity gradient. This equilibrium results from modification of the unstable radiative equilibrium jet and from eddy-induced dissipation, which has at equilibrium the value $r_{e}=0.2141$. The magnitude, spectra, and structure of the fluxes are realistic (DelSole and Farrell 1996; Zurita-Gotor 2007). The upgradient momentum flux forcing the jet is primarily concentrated in the upper layer in agreement with turbulence simulations (Thompson and Young 2007) and in contrast to scaling assumptions for diffusion (Lapeyre and Held 2003). The eddy energy has a maximum at global wavenumber 6 . The flow is stable at this wavenumber when the added dissipation associated with the turbulent fluctuations is included [cf. maximum at wavenumber 5 in Whitaker and Barcilon (1995)].

We now investigate how this equilibrium changes as a function of $\Delta T$. The upper- and lower-layer jet maxima are shown in Fig. 2. As $\Delta T$ increases, the upper jet maximum increases until $\Delta T=57 \mathrm{~K}$ over the $12 \times 10^{3}-\mathrm{km}$ channel, at which point the barotropic component of the mean jet collapses and the flow approaches the meridionally uniform jet associated with this temperature difference. ${ }^{4}$

\footnotetext{
${ }^{4}$ Because of the periodic boundary conditions imposed on the channel walls, the maintained meridional temperature difference remains equal to that imposed.
} 

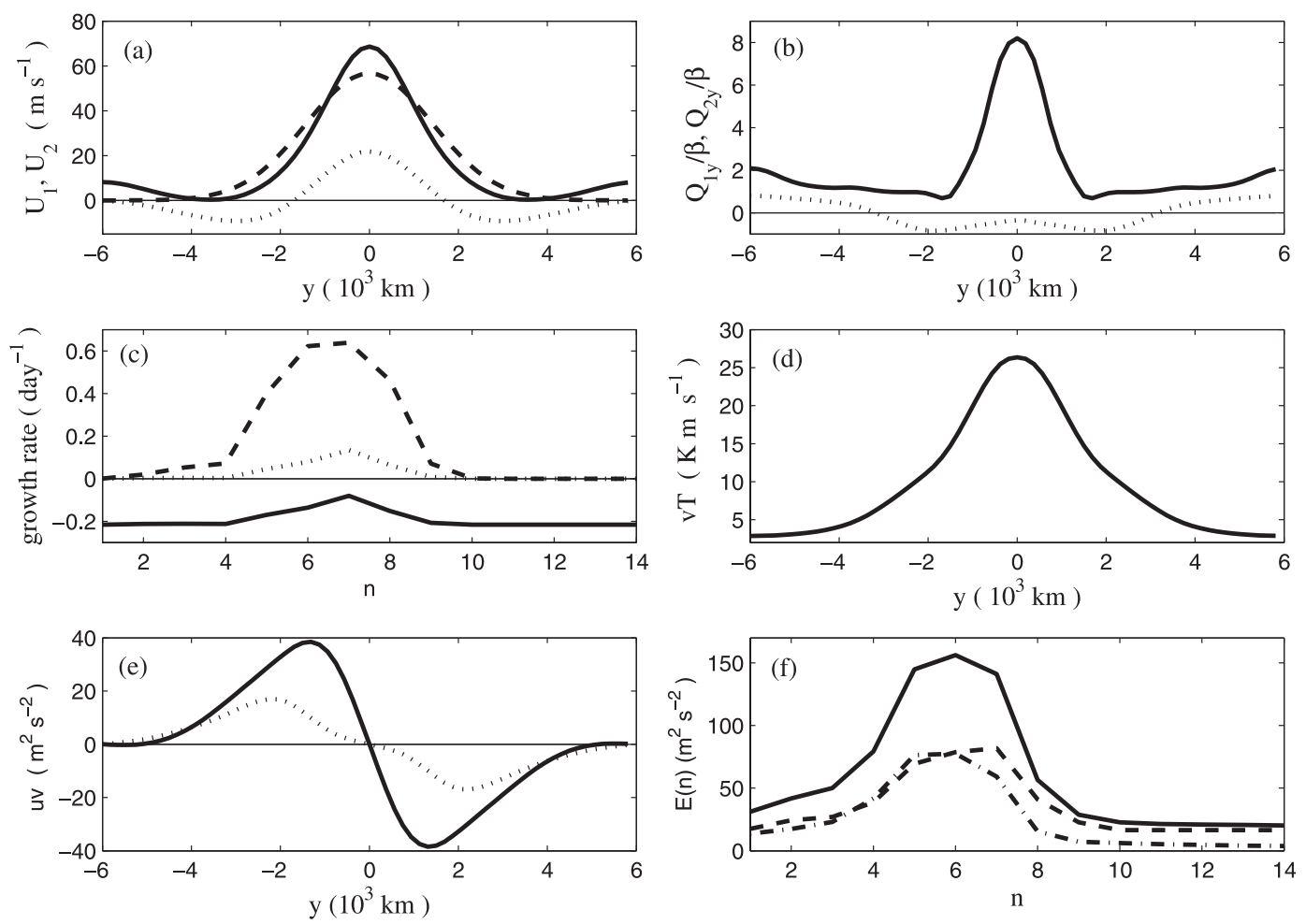

FIG. 1. (a) Meridional structure of the equilibrium upper- (solid) and lower-layer (dotted) jets maintained by the stochastic turbulence model in a channel relaxed toward the Gaussian jet (32) with $\Delta T=35 \mathrm{~K} /\left(12 \times 10^{3} \mathrm{~km}\right)$ (dashed). (b) The potential vorticity gradient of the two layers $Q_{1 y} / \beta$ (solid) and $Q_{2 y} / \beta$ (dotted). (c) The corresponding maximal modal growth rate of the radiative equilibrium jet (dashed), the equilibrated jet without the eddyinduced Rayleigh friction (dotted), and the equilibrated flows with the eddy-induced Rayleigh friction (solid), as a function of global wavenumber. The eddy dissipation coefficient is $r_{e}=0.2141 \mathrm{day}^{-1}$. (d) The meridional structure of the heat flux $\left(\mathrm{K} \mathrm{m} \mathrm{s}^{-1}\right.$ ). (e) The momentum flux in the upper (solid) and lower layer (dotted). (f) The total eddy energy per unit mass (solid), the eddy kinetic energy (dashed), and the eddy available potential energy (dasheddotted) as a function of global zonal wavenumber.

Collapse of the barotropic component of the jet at $\Delta T=$ $57 \mathrm{~K}$ implies corresponding stability and damping parameters $\beta L_{R}^{2} / \max \left(U_{R}^{-}\right)=0.35$ and $r_{2} L_{r} / \max \left(U_{R}^{-}\right)=$ 0.05 , which are close to the values for a similar state transition found by Thompson and Young (2007) (cf. their Fig. 11). The eddies in this regime are highly energetic, with $v_{\text {rms }}$ exceeding the mean jet speed. The eddy energy in this regime increase as $(\Delta T)^{5}$ whereas the heat flux increases as $(\Delta T)^{4}$, giving a higher-order diffusivity coefficient growing as $(\Delta T)^{3}$, as in the diffusive theory of Held and Larichev (1996) (shown in Fig. 3). In this limit the turbulence equilibrates a meridionally uniform jet that is made stable by eddy dissipation.

These dissipative equilibria characterize high- $\Delta T$ regimes, whereas for realistic midlatitude $\Delta T$ the equilibria take the form of jets, the eddy damping is subdominant in its contribution to equilibration, the upper-level momentum flux dominates the jet modification, and the heat flux increases approximately as $(\Delta T)^{3}$.

\section{b. Turbulent equilibration of a radiatively forced meridionally uniform shear}

Consider now a flow relaxed to a meridionally uniform baroclinic shear. We consider a channel of width $L_{y}=24$ to accommodate multiple jets and minimize the effects of channel size on the equilibria. All calculations are performed with the eddy field consisting of global zonal wavenumbers 1-14 and with damping parameters $r_{2}=$ $1 / 5 \mathrm{day}^{-1}$ and $r_{R}=1 / 10 \mathrm{day}^{-1}$. For the calculation of the mean eddy scale $L_{\text {eddy }}$, we assume nondimensional values $E_{0}=1$ and $\Sigma_{k} \operatorname{trace}\left(\mathbf{M}_{k} \mathbf{Q}_{k}\right)=0.1881$. Turbulence is maintained when the temperature difference across the channel is supercritical, the critical temperature difference for the assumed dissipation parameters being $\Delta T_{c}=31.9$ over half the channel (i.e., $L y=12$ ).

Equilibria as a function of the scattering efficiency $\epsilon$ show the same insensitivity to this parameter noted in the previous section in which the flow was relaxed to a localized jet. When the flow is relaxed, as here, to a 


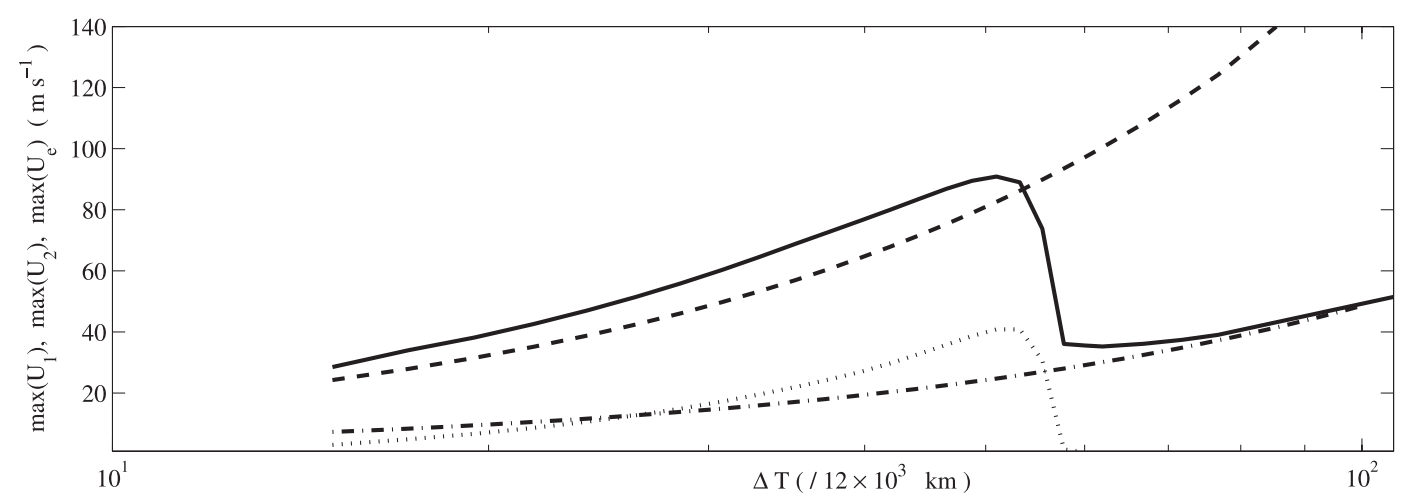

FIG. 2. Maximum upper-layer jet velocity (solid) and maximum lower-layer jet velocity (dotted) as a function of the temperature difference across the channel. The dashed line indicates the maximum shear of the radiative equilibrium jet; the dashed-dotted line is the upper-level velocity of the meridionally uniform flow associated with temperature difference $\Delta T$.

meridionally uniform shear, multiple jets form, with the number of jets depending on the supercriticality of the flow (cf. Farrell and Ioannou 2008).

Multiple jet equilibria maintained in a channel with mean thermal forcing $\Delta T=45 \mathrm{~K} /\left(12 \times 10^{3} \mathrm{~km}\right)$ and scattering coefficient $\epsilon=1$ corresponding to $r_{e}=0.1248$ day $^{-1}$ are shown in Fig. 4. These jets are equilibrated to a marginally stable state with stability due in part to the induced eddy dissipation and in part to modification of the jet structure. The associated fluxes of momentum and heat as well as the energy spectra shown in Fig. 4 are realistic.

For low supercriticality there exist many stable equilibria differing in meridional wavenumber. As the temperature contrast increases only the gravest meridional wavenumber jets survive. The maximum upper- and lower-level flows for the equilibrium configuration corresponding to meridional wavenumber 3 are shown as a function of $\Delta T$ in Fig. 5. As $\Delta T$ increases, the jet maximum increases until at $\Delta T=57.5$ jets can no longer be maintained and the flow collapses to a meridionally uniform flow (dashed line) stabilized by turbulent dissipation, as occurred in the case of the isolated jet described in the previous section.

\section{Conclusions}

Understanding turbulence remains a fundamental problem in fluid dynamics. The two-layer baroclinic model provides an analytically and computationally convenient system in which to study the dynamics of turbulence. In this work we described a closure that incorporates with maximum simplicity the essential dynamics of baroclinic turbulence: maintenance of variance by transient perturbation growth, replenishment of the transiently growing subspace by nonlinear energetically conservative eddyeddy scattering, and equilibration to a statistically steady state by a combination of nonlinear eddy-induced mean jet modification and eddy dissipation. These dynamics are modeled as follows: for the replenishment of the growing subspace a quadratic in velocity, white in time and space and conservative of energy parameterization of eddy-eddy scattering is used; an STM is used for the eddy fluxes and a SSST model for the equilibration. Despite the extremely simplified parameterization used to isolate the essential elements of turbulent dynamics, for radiative forcing appropriate for the midlatitude jets this closure predicts modification at turbulent equilibrium of a meridionally localized unstable jet to a marginally stable jet with a structure in agreement with

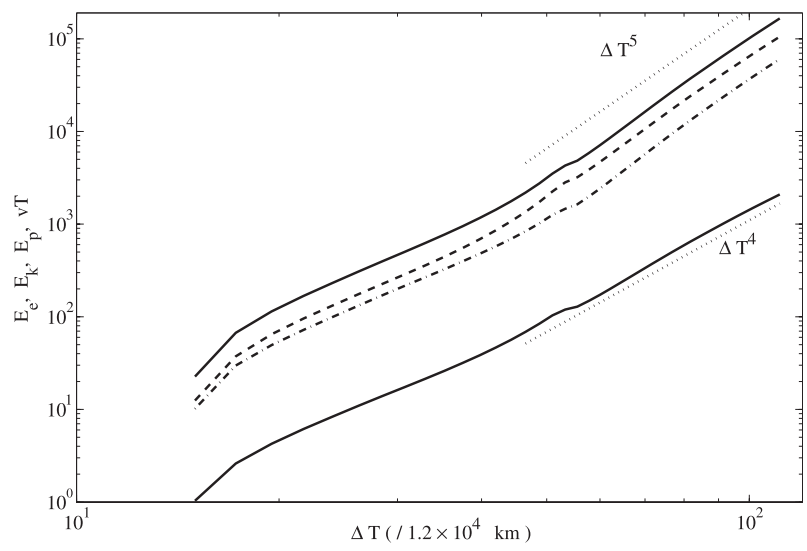

FIG. 3. Maximum heat flux $\left(\mathrm{K} \mathrm{m} \mathrm{s}^{-1}\right)$, which increases as $(\Delta T)^{4}$, implying that the diffusivity increases as $(\Delta T)^{3}$ (bottom solid). Maximum total eddy energy (top solid), maximum eddy kinetic energy (dashed), and maximum potential energy (dashed-dotted) $\left(\mathrm{m}^{2} \mathrm{~s}^{-2}\right)$ as a function of $\Delta T$. These quantities increase as $(\Delta T)^{5}$. 

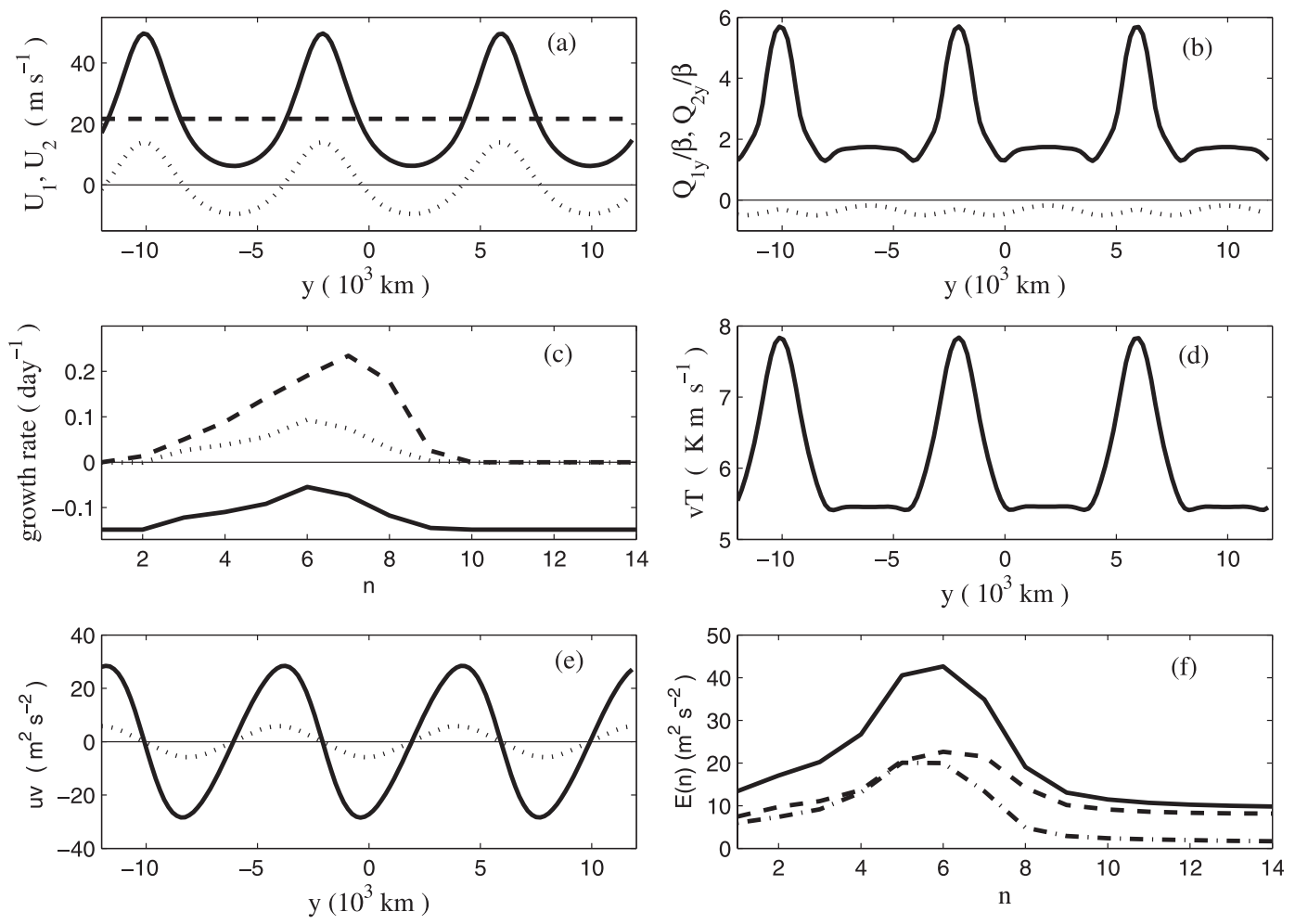

FIG. 4. (a) Meridional structure of the equilibrium upper- (solid) and lower-layer (dotted) jets maintained in a channel with $\Delta T=45 \mathrm{~K} /\left(12 \times 10^{3} \mathrm{~km}\right)$. The mean flow is relaxed toward a meridionally uniform shear (dashed). (b) The potential vorticity gradient of the two layers $Q_{1 y} / \beta$ (solid) and $Q_{2 y} / \beta$ (dotted). (c) The corresponding maximal modal growth rate of the imposed flow (dashed), the equilibrated flows without the eddy-induced Rayleigh friction (dotted), and the equilibrated flows with the eddy-induced Rayleigh friction, as a function of global wavenumber. The eddy dissipation coefficient is $r_{e}=0.1248$ day $^{-1}$ and the scattering coefficient $\epsilon=1$. (d) Meridional structure of the heat flux $\left(\mathrm{K} \mathrm{m} \mathrm{s}^{-1}\right)$. (e) The associated upper- (solid) and lower-level (dotted) momentum flux $\left(\mathrm{m}^{2} \mathrm{~s}^{-2}\right)$. (f) The total eddy energy per unit mass (solid), the eddy kinetic energy (dashed), and the eddy available potential energy (dasheddotted) as a function of global zonal wavenumber.

nonlinear simulations. This closure also predicts in the case of a wide channel the emergence of marginally stable multiple jets from a meridionally constant unstable thermal gradient. As the thermal gradient is increased across a wide channel, these multiple jets adjust to maintain marginally stable equilibria and the average heat flux increases rapidly with the imposed thermal gradient. This behavior agrees with the concept of baroclinic adjustment in the sense that the system is adjusted to stability, but the functional dependence of the heat flux on the thermal gradient varies to good approximation as a power law in agreement with predictions of higher-order thermal diffusion (Held and Larichev 1996; Pavan and Held 1996; Thompson and Young 2007). However, over the range of radiative forcing appropriate for midlatitude jets, the mechanism producing this powerlaw relation between eddy thermal flux and mean thermal gradient is baroclinic adjustment (i.e., equilibration to marginal stability) of multiple jets rather than thermal diffusion. Such power-law relations between quadratic quantities (such as the heat flux) and parameters influencing stability (such as the mean temperature gradient) are a general property of turbulence maintained by nonnormal perturbation growth processes (Farrell and Ioannou 2003, 2008). Moreover, whereas baroclinic adjustment only predicts adjustment to marginal stability and not the particular marginal stable state, SSST predicts the unique state obtained at statistical equilibrium and these unique states provide a theory for the general circulation of baroclinically turbulent atmospheres.

At very high thermal forcing the closure predicts that the (multiple) jet regime is replaced by a meridionally uniform regime in which stabilization occurs through eddy dissipation rather than by mean flow modification. 


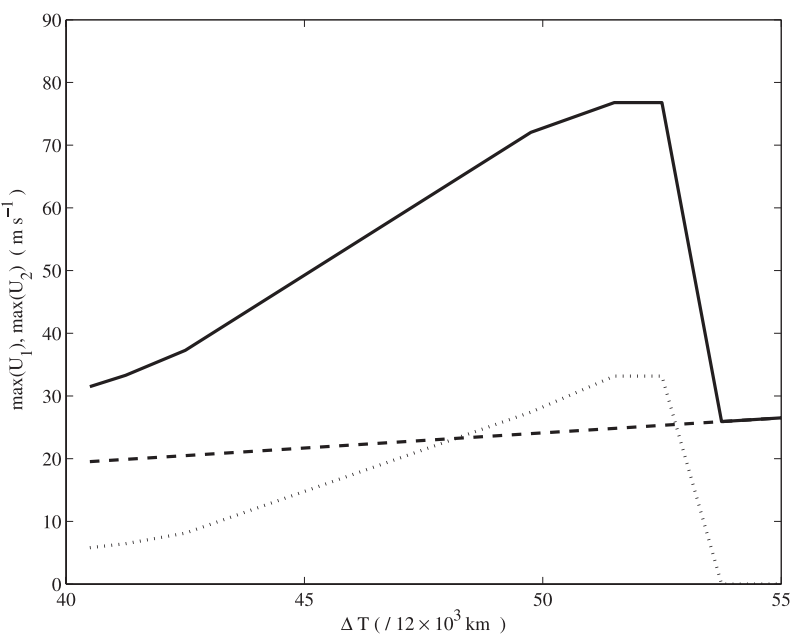

FIG. 5. Maximum upper-layer jet velocity (solid) and maximum lower-layer jet velocity (dotted) as a function of the temperature difference across the channel. The dashed line indicates the shear of the radiative equilibrium jet.

This model provides a physically correct closure for baroclinic turbulence that is optimally simplified to include only the essential elements of turbulent dynamics. It predicts observed turbulent equilibria and associated turbulent fluxes and the emergence, equilibration, and structure of the zonal jets associated with the turbulent state.

Acknowledgments. This work was partially supported by NSF ATM-0736022. Petros Ioannou has been partially supported by Kapodistrias Grant 70/4/5797, a program of the University of Athens.

\section{REFERENCES}

DelSole, T., 1996: Can quasigeostrophic turbulence be modeled stochastically? J. Atmos. Sci., 53, 1617-1633.

- 2001: A theory for the forcing and dissipation in stochastic turbulence models. J. Atmos. Sci., 58, 3762-3775.

_ 2004a: The necessity of instantaneous optimals in stationary turbulence. J. Atmos. Sci., 61, 1086-1091.

- 2004b: Stochastic models of quasigeostrophic turbulence. Surv. Geophys., 25, 107-194.

_ 2007: Optimal perturbations in quasigeostrophic turbulence. J. Atmos. Sci., 64, 1350-1364.

__ , and B. F. Farrell, 1995: A stochastically excited linear system as a model for quasigeostrophic turbulence: Analytic results for one- and two-layer fluids. J. Atmos. Sci., 52, 2531-2547.

— and — 1996: The quasi-linear equilibration of a thermally maintained, stochastically excited jet in a quasigeostrophic model. J. Atmos. Sci., 53, 1781-1797.

Farrell, B. F., 1982: The initial growth of disturbances in a baroclinic flow. J. Atmos. Sci., 39, 1663-1686.
1985: Transient growth of damped baroclinic waves. J. Atmos. Sci., 42, 2718-2727.

— 1989: Optimal excitation of baroclinic waves. J. Atmos. Sci., 46, 1193-1206.

— exhibits universality. Phys. Fluids, 5, 2298-2300.

$\ldots$, and 1993b: Stochastic dynamics of baroclinic waves. J. Atmos. Sci., 50, 4044-4057.

$\longrightarrow$, and — 1994: A theory for the statistical equilibrium energy spectrum and heat flux produced by transient baroclinic waves. J. Atmos. Sci., 51, 2685-2698.

$\longrightarrow$, and - 1995: Stochastic dynamics of the midlatitude atmospheric jet. J. Atmos. Sci., 52, 1642-1656.

— operators. J. Atmos. Sci., 53, 2025-2040.

$\longrightarrow$, and — 1996b: Generalized stability. Part II: Nonautonomous operators. J. Atmos. Sci., 53, 2041-2053.

, and - 2003: Structural stability of turbulent jets. J. Atmos. Sci., 60, 2101-2118.

, and - 2007: Structure and spacing of jets in barotropic turbulence. J. Atmos. Sci., 64, 3652-3665.

$\longrightarrow$, and — 2008: Formation of jets by baroclinic turbulence. J. Atmos. Sci., 65, 3353-3375.

$\longrightarrow$, and - 2009: Emergence of jets from turbulence in the shallow-water equations on an equatorial beta plane. J. Atmos. Sci., in press.

Gierasch, P. J., and Coauthors, 2000: Observation of moist convection in Jupiter's atmosphere. Nature, 403, 628-630.

Green, J. S. A., 1970: Transfer properties of the large-scale eddies and the general circulation of the atmospheres. Quart. J. Roy. Meteor. Soc., 96, 157-185.

Held, I. M., and V. D. Larichev, 1996: A scaling theory for horizontally homogeneous, baroclinically unstable flow on a beta plane. J. Atmos. Sci., 53, 946-952.

Ingersoll, A. P., P. J. Gierasch, D. Banfield, and A. R. Vasavada, 2000: Moist convection as an energy source for the largescale motions in Jupiter's atmosphere. Nature, 403, 630632.

Lapeyre, G., and I. M. Held, 2003: Diffusivity, kinetic energy dissipation, and closure theories for the poleward eddy heat flux. J. Atmos. Sci., 60, 2907-2916.

Newman, M., P. D. Sardeshmukh, and C. Penland, 1997: Stochastic forcing of the wintertime extratropical flow. J. Atmos. Sci., 54, 435-455.

Panetta, R. L., 1993: Zonal jets in wide baroclinically unstable regions: Persistence and scale selection. J. Atmos. Sci., 50, 2073-2106.

Pavan, V., and I. M. Held, 1996: The diffusive approximation for eddy fluxes in baroclinically unstable jets. J. Atmos. Sci., 53, 1262-1272.

Pedlosky, J., 1972: Limit cycles and unstable baroclinic waves. J. Atmos. Sci., 29, 53-63.

, 1977: A model of wave amplitude vacillation. J. Atmos. Sci., 34, 1898-1912.

— , and C. Frenzen, 1980: Chaotic and periodic behavior of finite-amplitude baroclinic waves. J. Atmos. Sci., 37, 11771196.

Sardeshmukh, P. D., and P. Sura, 2007: Multiscale impacts of variable heating in climate. J. Climate, 20, 5677-5695.

Stone, P. H., 1978: Baroclinic adjustment. J. Atmos. Sci., 35, 561-571.

Thompson, A. F., and W. R. Young, 2007: Two-layer baroclinic eddy heat fluxes: Zonal flows and energy balance. J. Atmos. Sci., 64, 3214-3231. 
Tung, K. K., and W. W. Orlando, 2003: The $k^{-3}$ and $k^{-5 / 3}$ energy spectrum of atmospheric turbulence: Quasigeostrophic twolevel model simulation. J. Atmos. Sci., 50, 824-835.

Valis, G. K., 2006: Atmospheric and Oceanic Fluid Dynamics: Fundamentals and Large-Scale Circulation. Cambridge University Press, $745 \mathrm{pp}$.

Whitaker, J. S., and A. Barcilon, 1995: Low-frequency variability and wavenumber selection in models with zonally symmetric forcing. J. Atmos. Sci., 52, 491-503.
—, and P. D. Sardeshmukh, 1998: A linear theory of extratropical synoptic eddy statistics. J. Atmos. Sci., 55, 237-258.

Zhang, Y., and I. M. Held, 1999: A linear stochastic model of a GCM's midlatitude storm tracks. J. Atmos. Sci., 56, 3416-3435.

Zurita-Gotor, P., 2007: The relation between baroclinic adjustment and turbulent diffusion in the two-layer model. J. Atmos. Sci., 64, 1284-1300. 